\title{
EDITORIAL \\ LA NORMALIDAD ROTA. IMPACTOS SOCIALES Y CULTURALES DE LA PANDEMIA DE COVID-19
}

Cómo citar: Ulloa Hung, J. (2021). Editorial. La normalidad rota. Impactos sociales y culturales de la pandemia de COVID-19. Ciencia y Sociedad, 46(2), 3-5. DOI: https://doi.org/10.22206/cys.2021.v46i2.pp3-5

Durante el último año y medio lo que pudiera considerarse nuestra "vida normal" o normalidad ha sido significativamente transformada por los complejos y disímiles efectos de la pandemia de coronavirus o COVID-19. Diversas esferas de la sociedad, la cultura, la educación, la salud, las políticas públicas, los hábitos alimentarios, los mercados laborales y las formas de socialización han sido objeto de importantes impactos y transformaciones, al mismo tiempo que términos y frases como "distanciamiento social", "autoaislamiento", "aplanamiento de la curva", "cuarentena", "toque de queda", "contagio", "brote", "propagación" han tenido, como nunca, una presencia cotidiana en nuestro vocabulario y discurso.

En este número 2 del 2021, Ciencia y Sociedad dedica una edición especial a los impactos sociales y culturales de la pandemia de COVID 19, contemplando diversos enfoques, disciplinas y campos sociales. Aunque el conjunto de artículos que comprende este número está centrado en los contextos de República Dominicana y México, las problemáticas tratadas tienen un alcance mayor, y abarcan situa- ciones, experiencias, o repercusiones similares en otros países de América Latina y el mundo.

Este número especial de Ciencia y Sociedad inicia con un análisis de uno de los aspectos donde se ha manifestado de manera más significativa el impacto de la pandemia COVID-19, la conducta alimentaria y el consumo de alcohol, fundamentalmente motivados por las restricciones impuestas a nuestra socialización por medidas de reclusión o aislamiento. A través de un estudio con diseño transversal exploratorio y correlacional, esta primera colaboración analiza el impacto de las emociones y del estrés generado por la pandemia en la ingesta de comidas y bebidas alcohólicas, e identifica variables psicosociales asociadas a esas conductas. En ese orden, el artículo es capaz de esbozar con carácter predictivo algunos de los factores vinculados a los consumos de ambos elementos, así como la importancia del apoyo social como un factor protector de los sujetos. Ambos aspectos configuran uno de los aportes más relevantes del artículo, en tanto la identificación de las variables psicosociales asociadas a estos consumos 
constituyen un elemento de peso para diseñar estrategias de intervención, encaminadas a mitigar los efectos de las situaciones altamente estresantes de la crisis sanitaria sobre nuestra conducta alimentaria y nuestro consumo de bebidas alcohólicas.

Por su parte, los efectos de la pandemia de COVID-19 en el contexto social dominicano se exponen a través de dos artículos en este número de Ciencia y Sociedad. El primero de ellos presenta los resultados de un estudio que cuantifica los cambios y transformaciones en el perfil de los trabajadores requeridos en la República Dominicana durante el último año, especialmente a partir de los efectos y cambios generados por la pandemia. A partir de un análisis longitudinal de la demanda de personal de las cinco agencias de empleo con mayor número de avisos web en el país, esta colaboración establece la relación entre la demanda de puestos vacantes, los sectores económicos más impactados por la pandemia y otros requerimientos sociales como grupos de edades, el género y las habilidades y destrezas requeridas. Sus resultados no solo constituyen un llamado de atención sobre las transformaciones generadas por el COVID-19 en el sector laboral dominicano, además reflejan la clara necesidad de realizar ajustes en los programas y modelos educativos en relación con estas nuevas transformaciones, así como la adopción de políticas públicas que incentiven el empleo en los grupos poblacionales más vulnerables.

La fuerza laboral dominicana, desde la perspectiva de su salud mental, también es objeto de atención en este número de Ciencia y Sociedad, fundamentalmente en relación con sus niveles de riesgo de contagio de COVID-19. El artículo intitulado "Burnout, empatía y grados de exposición al COVID-19 en empleados dominicanos" refleja los resultados de un estudio que, desde una perspectiva correlacional, intenta establecer los vínculos entre estas tres variables tomando en consideración aspectos sociodemográficos externos. Su objetivo esencial, además de identificar las potenciales rela- ciones entre grados de exposición al COVID-19 y el síndrome de Burnout, contempla dos tipos de empatía, la cognitiva y la afectiva. Utilizando herramientas de medición básicamente cuantitativas, y a partir de un muestreo no probabilístico, esta colaboración presenta la ausencia de correlaciones entre dos de las variables analizadas: burnout, ingresos económicos y empatía, en sus dos variantes estudiadas. Sin embargo, asimismo muestra la correlación positiva y significativa entre empatía afectiva y niveles de exposición al COVID-19 en los profesionales de la salud. Este último aspecto señala, abiertamente, la necesidad de evaluar el burnout para mitigar sus efectos negativos sobre determinados grupos poblacionales, en especial entre los profesionales de la salud.

Las miradas hacia los impactos de la pandemia sobre regiones concretas aparecen desplegadas en esta edición de Ciencia y Sociedad a partir de colaboraciones que evalúan dos elementos relevantes: el accionar de organizaciones gubernamentales y no gubernamentales, así como el impacto sociocultural de medidas administrativas adoptadas para enfrentar el COVID-19. Al respecto, Tamaulipas y Puebla, dos regiones mexicanas, constituyen el centro de atención de estos artículos y su estudio.

El análisis del accionar de organizaciones gubernamentales y no gubernamentales en la atención a migrantes durante el comienzo de la pandemia de COVID-19 en la frontera de Tamaulipas, noreste mexicano, constituye una evaluación de las políticas y acciones públicas para la identificación de problemáticas y elaboración de respuestas frente a los embates de esta enfermedad. Un aspecto relevante de esa evaluación es la identificación de las sinergias y conflictos que emergieron durante este proceso, así como la exposición de experiencias que pueden constituir puntos de partida y referencias importantes para mejorar ese tipo de acciones en el futuro y en otros contextos con actividad migratoria relevante. 
El impacto de medidas administrativas frente al COVID-19 en dinámicas socioculturales del poblado de Cuetzalan del Progreso, Puebla, conforma el otro ejemplo significativo de los efectos de medidas y políticas públicas sobre determinados sectores sociales y económicos, en especial sobre poblaciones con actividad económica altamente dependiente del turismo. Este artículo tiene además la particularidad de develar esas implicaciones sobre un grupo cuyo núcleo poblacional está compuesto por población originaria (masewal) con amplios niveles de marginación socioeconómica. Utilizando métodos etnográficos esta colaboración analiza carencias de las decisiones tomadas por las autoridades para evitar contagios por COVID-19, especialmente centrando la atención en dos elementos: la ausencia de participación comunitaria y de la valoración de su alta dependencia del turismo. En ese mismo sentido, el artículo también refleja cómo la resiliencia territorial constituyó un elemento alternativo y de peso en el diseño de estrategias comunitarias que contribuyeron al fortalecimiento social en el enfrentamiento de esta situación.

Un último grupo de trabajos incluidos en esta entrega especial de Ciencia y Sociedad constituyen abordajes más generales sobre renglones sociales y económicos impactados por la pandemia de COVID-19. Por un lado, el entorno mexicano y el sector laboral asociado al turismo vuelven a constituirse en el escenario de este tipo de estudios. Por el otro, una especie de balance general de lo que ha implicado la pandemia para ese país se presenta como uno de los temas centrales tratados en estas colaboraciones.

Las perspectivas laborales en el sector turístico mexicano post-COVID-19 son analizadas a la luz de antecedentes sobre la precariedad laboral de ese sector económico, previos a la pandemia, y cómo estos factores se han agravado ante la contingencia sanitaria. A partir de este análisis, el artículo esboza algunas de las situaciones que enfrentará la recuperación de este sector, con énfasis particular en aspectos o cuestiones de orden contractual, tomando en consideración diferentes factores laborales asociados a esta industria.

Finalmente, una revisión de las implicaciones más sobresalientes generadas por la pandemia de COVID-19 en espacios de la vida social mexicana después de un año, evalúa aquellos que constituyen potenciales escenarios de contagio y los que pueden transformarse en modelos de prevención y control de la enfermedad. El aspecto más relevante de esta especie de balance sobre los impactos sociales creados por la emergencia sanitaria del COVID-19 son las observaciones sobre los efectos en la vida cotidiana y, a su vez, la capacidad de mostrar que la llamada "ruptura de la normalidad" es en el fondo una nueva revelación de la amplia desigualdad social que padecen los países de América Latina y el Caribe.

\section{Dr. Jorge Ulloa Hung}

Profesor Investigador del Instituto Tecnológico de

Santo Domingo (INTEC)

Director de Ciencia y Sociedad

Correo-e: jorge.ulloa@intec.edu.do

Página web: https://www.intec.edu.do 